RESUMEN METEOROLÓGICO AÑO $2012^{1}$

ESTACIÓN "JORGE C. SCHYTHE"

(53ํำ' S; 705'W; 6m.s.n.m)

METEOROLOGICAL SUMMARY YEAR 2012, "JORGE C. SCHYTHE” STATION ${ }^{1}$

Nicolás Butorovic ${ }^{2}$

La información corresponde a datos recolectados en la estación climática Jorge C. Schythe, ubicada en el campus del Instituto de la Patagonia de la Universidad de Magallanes. Esta estación cuenta con instrumental meteorológico tradicional y automático, y opera en convenios con la Dirección Meteorológica de Chile (DMC), la Dirección General de Aguas (DGA) y la Patagonia Research Fundation (PRF).

La temperatura se midió con termómetros de mercurio normales, un termómetro de mercurio de máxima y un termómetro de alcohol etílico con testigo para la temperatura mínima. Las mediciones las efectuó un observador en forma diaria durante todo el año, a las horas correspondientes a la observación. Para esta variable, también se contó con un higrotermógrafo que registra la temperatura y la humedad en forma simultánea y continua, de manera que queda un registro de las oscilaciones de estas variables en el tiempo. Se dispuso también, dentro del cobertizo meteorológico, de un termómetro de mercurio de bulbo húmedo para el registro de la temperatura del aire saturado. Con esta variable se obtiene y se le da precisión a la medida de la humedad relativa.

La precipitación se midió con un pluviómetro tipo Hellman y además se registró en un pluviógrafo del mismo tipo. La evaporación se midió en un estanque tipo A pan y junto con la precipitación, esta medición se hizo diariamente a la 8:00 horas local de invierno (12 UTC). Las horas de sol se registran mediante un heliógrafo.

La radiación global (directa más difusa) se midió con un actinógrafo de placa bi-metálica, cuyo rango de registro, dentro del espectro electromagnético solar, está comprendido entre los $0,35 \mu \mathrm{m}$ y los $3 \mu$ $\mathrm{m}$ aproximadamente y corresponde a la radiación global recibida en una superficie horizontal.

El viento se midió con un anemógrafo Belfor, ubicado a $10 \mathrm{~m}$ s.n.s., el cual registra en

1 Corresponde a Proyecto F3-01G-97 "Programa de Información y Documentación Climática".

${ }^{2}$ Área de Geociencias, Instituto de la Patagonia, Universidad de Magallanes, Punta Arenas, Chile.nicolas.butorovic@umag.cl 
forma continua la velocidad y la dirección. El registro muestra detalles de las variaciones de este vector en su velocidad y dirección en forma simultánea, pudiendo apreciarse con una exactitud aceptable la hora de los eventos instantáneos importantes, como las rachas máximas diarias, duración de temporales de viento, etc. De aquí se extrae el valor medio horario y la velocidad predominante en esa hora, conformando así una serie anual de tiempo de unos 8.760 datos de velocidad e igual número de datos de dirección.

\section{Parámetros medidos}

A. TEMPERATURAS. El promedio para el 2012 fue de $6.4^{\circ} \mathrm{C}$, valor inferior en $0.1^{\circ} \mathrm{C}$ al promedio normal para Punta Arenas (Zamora \& Santana 1979, Endlicher \& Santana 1988, Santana et al. 2009) e inferior en $0.5^{\circ} \mathrm{C}$ al promedio obtenido el 2011 (Butorovic 2012). El mes más cálido fue enero, con una temperatura media de $11.9^{\circ} \mathrm{C}$, valor superior en $0.9^{\circ} \mathrm{C}$ al valor medio normal de este mes. Por su parte, el mes más frío fue junio con un promedio de $1.6^{\circ} \mathrm{C}$, siendo este valor inferior en $0.6^{\circ} \mathrm{C}$ al compararlo con el promedio normal. No obstante, el mes que presentó la mayor diferencia respecto al valor normal fue febrero con una disminución de $2.2^{\circ} \mathrm{C}$. Otro mes que presentó una baja en su promedio fue diciembre con $1.5^{\circ} \mathrm{C}$ menos; un valor de $8.7^{\circ} \mathrm{C}\left(10.2^{\circ} \mathrm{C}\right.$ es el promedio normal).

De los meses que presentaron un alza de temperatura media respecto a su valor normal correspondiente independiente del mes de enero, fueron marzo, julio, agosto y septiembre con alzas de $1.1^{\circ} \mathrm{C}, 0.5^{\circ} \mathrm{C}, 1.0^{\circ} \mathrm{C}$ y $0.4^{\circ} \mathrm{C}$ respectivamente. $\mathrm{Si}$ bien estas variaciones de los promedios mensuales alteraron el promedio anual de la ciudad de Punta Arenas, es bueno detenerse a analizar el bajo promedio registrado durante los meses de febrero y diciembre.

El promedio obtenido en febrero fue de $8.4^{\circ} \mathrm{C}$ valor inferior en $2.2^{\circ} \mathrm{C}$ al valor normal histórico para este mes que es de $10.6^{\circ} \mathrm{C}$; desde el año 1972 para el mes de febrero no se registraba una temperatura media tan baja; en ese año la media fue igual $7.2^{\circ} \mathrm{C}$, lo que constituye una excepción a la regla para un mes de estación de verano; a su vez diciembre presentó una media de $8.7^{\circ} \mathrm{C}$ valor inferior en $1.5^{\circ} \mathrm{C}$ al compararlo con el promedio normal histórico $\left(10.2^{\circ} \mathrm{C}\right)$; igual desde 1991 que un mes de diciembre no era tan frío en que se registró

TABLA 1. Temperaturas $\left({ }^{\circ} \mathrm{C}\right)$

\begin{tabular}{lccccccccc}
\hline & Temp. & Max. & Min. & Med. & Med. & T & T & T & T. prom \\
& Media & Abs. & Abs. & Max. & Min. & $08: 00$ & $14: 00$ & $19: 00$ & $\begin{array}{c}1888- \\
2012\end{array}$ \\
\hline Enero & 11.9 & 21.3 & 0.3 & 16.6 & 7.2 & 11.8 & 14.7 & 12.1 & 11.0 \\
Febrero & 8.4 & 21.1 & -1.9 & 12.9 & 4.0 & 8.3 & 11.0 & 8.6 & 10.6 \\
Marzo & 10.0 & 18.3 & -0.8 & 13.3 & 7.1 & 9.5 & 12.0 & 10.2 & 8.9 \\
Abril & 5.7 & 14.1 & -5.1 & 9.6 & 2.2 & 5.3 & 8.0 & 5.7 & 6.5 \\
Mayo & 3.2 & 12.6 & -5.0 & 6.4 & 0.0 & 2.7 & 5.5 & 3.6 & 4.0 \\
Junio & 1.6 & 8.9 & -7.0 & 4.2 & -0.9 & 1.3 & 3.1 & 2.0 & 2.2 \\
Julio & 2.3 & 8.7 & -4.0 & 4.9 & -0.3 & 1.9 & 3.5 & 2.5 & 1.8 \\
Agosto & 3.8 & 10.8 & -4.7 & 7.5 & 1.0 & 2.9 & 6.0 & 3.7 & 2.8 \\
Septiembre & 5.1 & 11.4 & -2.0 & 8.7 & 1.9 & 4.9 & 7.2 & 4.9 & 4.7 \\
Octubre & 7.0 & 14.8 & -0.3 & 11.1 & 3.7 & 7.0 & 9.5 & 6.3 & 6.9 \\
Noviembre & 9.0 & 20.2 & -0.9 & 13.3 & 4.7 & 8.6 & 11.6 & 9.3 & 8.7 \\
Diciembre & 8.7 & 16.0 & 0.2 & 12.4 & 4.3 & 8.9 & 10.5 & 9.2 & 10.2 \\
\hline Promedio & 6.4 & 14.9 & -2.6 & 10.1 & 2.9 & 6.1 & 8.6 & 6.5 & 6.5 \\
\hline
\end{tabular}


una media de $7.9^{\circ} \mathrm{C}$ en ese año.

$\mathrm{Si}$ bien los promedios invernales se mantuvieron dentro de un rango normal, fueron los meses de otoño: marzo, abril y mayo los que presentaron un promedio bajo lo normal lo que trae una baja de la temperatura anual para 2012 en la ciudad de Punta Arenas.

Las medias anuales de las máximas y las mínimas diarias fueron $14.9^{\circ} \mathrm{C}$ y $-2.6^{\circ} \mathrm{C}$, en tanto que los promedios de las 8:00, 14:00 y 19:00 horas fueron de $6.1^{\circ} \mathrm{C}, 8.6^{\circ} \mathrm{C}$ y $6.5^{\circ} \mathrm{C}$ respectivamente.

La temperatura máxima absoluta alcanzó a los $21.3^{\circ} \mathrm{C}$ y se registró el día 04 de enero a las 12:00 horas, mientras que la mínima absoluta llegó a los $-7.0^{\circ} \mathrm{C}$, registrándose el 02 de junio a las 06:10 horas.

La última temperatura bajo cero grados del año 2011 ocurrió el 02 de octubre, llegando a $-2.9^{\circ} \mathrm{C}$ y la primera del 2012 se presentó el 18 de febrero alcanzando a $-1.9^{\circ} \mathrm{C}$, determinando así un período vegetativo libre de heladas de cinco meses aproximadamente (139 días). Por su parte, la última temperatura negativa de este año ocurrió el día 28 de noviembre, alcanzando a los $-0.9^{\circ} \mathrm{C}$.

La tabla 1 muestra los valores medios y extremos alcanzados en cada mes durante el 2012. La última columna de esta tabla muestra el promedio histórico de Punta Arenas.

TABLA 2. Humedad Relativa (\%)

\begin{tabular}{lcccccc}
\hline & Hum. & Med & Med & Med & Med. & Med. \\
& Med. & Max & Min & $08: 00$ & $14: 00$ & $19: 00$ \\
\hline Enero & 63.2 & 100.0 & 34.0 & 68.3 & 56.7 & 64.5 \\
Febrero & 62.9 & 100.0 & 37.0 & 70.4 & 58.9 & 59.2 \\
Marzo & 73.8 & 90.0 & 60.0 & 86.0 & 85.0 & 65.0 \\
Abril & 78.4 & 87.0 & 60.0 & 85.0 & 65.0 & 67.0 \\
Mayo & 81.2 & 90.0 & 64.0 & 77.0 & 64.0 & 76.0 \\
Junio & 76.9 & 90.0 & 76.0 & 82.0 & 79.0 & 88.0 \\
Julio & 79.1 & 96.0 & 72.0 & 92.0 & 76.0 & 88.0 \\
Agosto & 77.5 & 94.0 & 60.0 & 92.0 & 66.0 & 65.0 \\
Septiembre & 77.2 & 72.0 & 55.0 & 55.0 & 69.0 & 68.0 \\
Octubre & 65.0 & 82.0 & 60.0 & 60.0 & 64.0 & 76.0 \\
Noviembre & 64.6 & 92.0 & 68.0 & 83.0 & 90.0 & 76.0 \\
Diciembre & 68.5 & 93.0 & 50.0 & 90.0 & 50.0 & 72.0 \\
\hline Promedio & 72.4 & 90.5 & 58.0 & 78.4 & 68.6 & 72.1 \\
\hline
\end{tabular}

B. HUMEDAD RELATIVA. La humedad promedio del año 2012 fue del $72.4 \%$. El mes de mayor promedio fue mayo con un $81.2 \%$, mientras que el de menor humedad promedio correspondió a febrero con un $62.9 \%$. Las medias anuales de las máximas y mínimas diarias fueron de $90.5 \%$ y $58.0 \%$ respectivamente.

El promedio de las 8:00 horas llegó al 78.4 $\%$, el de las 14:00 fue del 68.6 \% y el de las 19:00 horas llegó al $72.1 \%$. El detalle mensual se muestra en la tabla 2.

C. PRECIPITACIONES. El total anual para el año
2012 alcanzó a los $638.1 \mathrm{~mm}$ ó lt/m² en 189 días que presentaron precipitaciones. Este monto es superior en $193.7 \mathrm{~mm}$ a la precipitación anual promedio de los últimos 124 años, cuyo valor medio alcanza a los $444.4 \mathrm{~mm}$. Con este monto y de acuerdo a la clasificación hecha por Santana (1984), para las lluvias anuales de Punta Arenas, el año 2012 queda clasificado como un año muy lluvioso.

Hay que destacar que por segundo año consecutivo la ciudad supera la barrera de los $600.0 \mathrm{~mm}$ anuales 
de precipitación, situación que no se había dado nunca en registros de 124 años para esta variable.

De este total anual alcanzado, $573.4 \mathrm{~mm}$ (equivalente a un $89.9 \%$ ) precipitaron en forma de agua y $32.9 \mathrm{~mm}$ (equivalentes al $5.2 \%$ ) lo hicieron en forma de agua-nieve. La precipitación de nieve (4.9\%) llegó a los $31.8 \mathrm{~cm}$ (equivalentes a igual cantidad de mm de agua), la que se presentó en los meses de mayo, junio y julio.

Cabe destacar que el mes de junio fue el que presentó la mayor precipitación de nieve del período invernal con un monto de $14.1 \mathrm{~cm}$; seguido de mayo con $8.9 \mathrm{~cm}$ y finalmente julio con $6.6 \mathrm{~cm}$. Como dato anexo se destaca que durante el mes de octubre en plena estación de primavera precipitó $1.2 \mathrm{~cm}$ el día 25.

Los meses que presentaron montos superiores a sus promedios normales fueron: marzo, abril, mayo, julio, septiembre, y diciembre. De estos, los ascensos más notables corresponden a diciembre, marzo, y mayo con diferencias positivas de $67.2 \mathrm{~mm}$; 58.7 mm y $45.9 \mathrm{~mm}$ respectivamente.
Dentro de esta alzas de las precipitaciones para el año 2012, sobresalen los meses de marzo y diciembre con montos idénticos de $102.4 \mathrm{~mm}$ para ambos meses; lo que representa un alza de $152 \%$ y $142 \%$ respectivamente. Si bien las precipitaciones en estos meses fueron altas, no están dentro de los record de sus respectivos meses.

Hay que destacar que en términos estadísticos estos dos meses hacen la diferencia alcanzada en la precipitación total del año 2012, los $638.1 \mathrm{~mm}$ caídos representan un alza del 229\% respecto a un año normal histórico para la ciudad de Punta Arenas.

Los meses de menor precipitación fueron febrero, agosto y noviembre, que registraron montos de $18.0 \mathrm{~mm}$; $21.7 \mathrm{~mm}$ y $24.0 \mathrm{~mm}$, respectivamente. La distribución mensual de la precipitación así como su tipo y los promedios mensuales históricos, se muestran en la tabla 3.

La máxima precipitación en un día alcanzó a los $50.8 \mathrm{~mm}$ y ocurrió en el día 11 de marzo; destaca también en su monto diario la precipitación de $34.1 \mathrm{~mm}$ registrada el día 12 del mismo mes.

TABLA 3. Precipitaciones y Evaporación

\begin{tabular}{lcccccc}
\hline Meses & $\begin{array}{c}\text { Agua } \\
(\mathrm{mm})\end{array}$ & $\begin{array}{c}\text { Agua-nieve } \\
(\mathrm{mm})\end{array}$ & $\begin{array}{c}\text { Nieve } \\
(\mathrm{cm})\end{array}$ & $\begin{array}{c}\text { Total } \\
(\mathrm{mm})\end{array}$ & $\begin{array}{c}\text { Prom } \\
1888 / 2012\end{array}$ & $\begin{array}{c}\text { Evapor. } \\
(\mathrm{mm})\end{array}$ \\
\hline Enero & 25.8 & 0.0 & 0.0 & 25.8 & 37.1 & 132.9 \\
Febrero & 18.0 & 0.0 & 0.0 & 18.0 & 31.1 & 115.3 \\
Marzo & 102.4 & 0.0 & 0.0 & 102.4 & 43.7 & 121.1 \\
Abril & 64.2 & 0.0 & 0.0 & 64.2 & 47.9 & 51.7 \\
Mayo & 66.2 & 17.6 & 8.9 & 92.7 & 46.8 & 26.6 \\
Junio & 17.8 & 3.4 & 14.1 & 35.3 & 37.5 & 0.0 \\
Julio & 31.2 & 7.8 & 6.6 & 45.6 & 36.1 & 51.9 \\
Agosto & 17.8 & 2.9 & 1.0 & 21.7 & 40.0 & 26.5 \\
Septiembre & 86.8 & 0.0 & 0.0 & 86.8 & 32.3 & 78.2 \\
Octubre & 16.8 & 1.2 & 1.2 & 19.2 & 27.5 & 141.3 \\
Noviembre & 24.0 & 0.0 & 0.0 & 24.0 & 29.2 & 129.3 \\
Diciembre & 102.4 & 0.0 & 0.0 & 102.4 & 35.2 & 130.7 \\
\hline Total & 573.4 & 32.9 & 31.8 & 638.1 & 444.4 & 1005.5 \\
\hline
\end{tabular}


TABLA 4. Precipitación diaria (mm).

\begin{tabular}{|c|c|c|c|c|c|c|c|c|c|c|c|c|}
\hline Dia & Ene & $\mathrm{Feb}$ & Mar & Abr & May & Jun & Jul & Ago & Sep & Oct & Nov & Dic \\
\hline 1 & - & - & 1.5 & - & 0.7 & 1.1 & 0.2 & - & - & - & 6.7 & 7.8 \\
\hline 2 & 0.9 & - & 1.3 & 12.9 & - & 0.9 & 1.4 & 0.4 & - & - & 2.1 & - \\
\hline 3 & - & 0.5 & 0.7 & 0.7 & 0.5 & 1.8 & 0.4 & - & - & - & - & - \\
\hline 4 & 4.2 & 2.7 & - & 3.4 & 1.8 & 1.7 & 0.2 & - & 1.9 & - & - & - \\
\hline 5 & - & 4.9 & - & - & 6.2 & - & - & - & 3.2 & - & - & 0.3 \\
\hline 6 & - & - & 1.2 & 1.7 & 1.3 & - & 3.4 & 0.9 & 8.7 & - & 3.6 & 1.8 \\
\hline 7 & - & 1.2 & 0.2 & 0.4 & - & - & 0.2 & 0.6 & 9.8 & 0.4 & 3.5 & - \\
\hline 8 & 1.2 & - & 0.2 & 0.5 & 1.5 & 1.7 & - & - & 16.9 & - & 1.7 & 0.9 \\
\hline 9 & 8.2 & - & - & 1.9 & 9.7 & 1.2 & 0.5 & 0.6 & 6.3 & 0.9 & - & - \\
\hline 10 & 0.5 & - & 7.2 & - & 0.9 & 0.2 & - & 0.8 & 0.5 & 4.7 & - & 8.9 \\
\hline 11 & - & - & 50.8 & 6.2 & 2.5 & - & 2.7 & 0.3 & - & - & - & - \\
\hline 12 & - & 2.1 & 34.1 & - & 3.6 & - & 0.1 & 0.4 & 6.2 & - & - & - \\
\hline 13 & 2.9 & 1.6 & 2.1 & 0.9 & 0.7 & - & 0.5 & 0.1 & 9.2 & - & - & 3.9 \\
\hline 14 & 0.6 & - & - & 2.7 & 0.2 & - & - & - & 8.2 & - & 0.8 & 7.8 \\
\hline 15 & - & - & - & 0.4 & 1.4 & - & 1.3 & - & 4.3 & - & - & 10.8 \\
\hline 16 & - & 1.1 & - & 10.9 & - & - & 0.6 & - & 0.5 & - & - & 6.9 \\
\hline 17 & - & - & 0.2 & 1.9 & - & 1.7 & 4.5 & 0.9 & - & - & - & - \\
\hline 18 & - & - & - & 1.4 & 1.9 & - & 4.9 & - & - & - & - & 27.6 \\
\hline 19 & - & 1.3 & - & 10.7 & 2.1 & 2.7 & 0.6 & 0.2 & - & - & - & 9.3 \\
\hline 20 & - & - & - & - & 4.2 & - & - & 4.5 & - & - & 1.4 & 3.4 \\
\hline 21 & - & - & - & - & 1.2 & 4.2 & 3.8 & 0.3 & 1.5 & - & 2.7 & 10.3 \\
\hline 22 & - & - & - & 2.7 & 8.4 & 4.2 & 1.2 & 1.8 & - & - & 0.2 & - \\
\hline 23 & - & - & - & 0.4 & 0.3 & - & - & 0.7 & - & 3.2 & - & - \\
\hline 24 & - & - & - & - & - & 10.5 & 8.2 & 0.4 & - & 0.8 & - & - \\
\hline 25 & 2.2 & 2.6 & - & - & 3.6 & - & 4.2 & 2.5 & - & 1.2 & 0.5 & - \\
\hline 26 & - & - & 1.7 & 0.3 & 10.4 & - & 0.8 & 3.5 & - & - & 0.8 & - \\
\hline 27 & 2.7 & - & - & 0.7 & 6.4 & 0.8 & 3.2 & 0.5 & - & 5.7 & - & 0.6 \\
\hline 28 & - & - & - & 1.2 & 23.2 & 0.4 & 2.2 & - & 0.6 & 2.3 & - & 2.1 \\
\hline 29 & 0.3 & & 0.6 & 1.4 & - & 1.9 & 0.5 & 0.7 & 6.7 & - & - & - \\
\hline 30 & 0.9 & & - & 0.9 & - & 0.3 & - & 1.6 & 2.3 & - & - & - \\
\hline 31 & 1.2 & & 0.6 & & - & & - & - & & - & & - \\
\hline Total & 25.8 & 18.0 & 102.4 & 64.2 & 92.7 & 35.3 & 45.6 & 21.7 & 86.8 & 19.2 & 24.0 & 102.4 \\
\hline Max $24 \mathrm{~h}$ & 8.2 & 4.9 & 50.8 & 12.9 & 23.2 & 10.5 & 8.2 & 4.5 & 16.9 & 5.7 & 6.7 & 27.6 \\
\hline № /días & 12.0 & 9.0 & 14.0 & 22.0 & 23.0 & 16.0 & 23.0 & 20.0 & 16.0 & 8.0 & 11.0 & 15.0 \\
\hline
\end{tabular}


Cabe destacar que durante los días 10 al 13 de marzo ocurrió al aluvión del río de las minas en que precipitaron en esos cuatro días la cantidad de $94.2 \mathrm{~mm}$; todo un record para la ciudad de Punta Arenas. El detalle diario de la precipitación se muestra en la tabla 4.

D. EVAPORACIÓN. El total de agua evaporada desde la superficie del suelo durante el año 2012, llegó a $1005.5 \mathrm{~mm}$, superando a las precipitaciones en más de $367.4 \mathrm{~mm}$. De este total, la mayor parte se evaporó en los meses de verano y primavera, evidenciando el carácter estacional de este parámetro. El valor alcanzado este año es inferior en $166.8 \mathrm{~mm}$ al compararlo con el año 2011. El mayor monto lo registró octubre, con $141.3 \mathrm{~mm}$, seguido de enero con un total de $132.9 \mathrm{~mm}$. Otros meses con montos de evaporación importantes y sobre 100 mm fueron febrero, marzo, noviembre y diciembre. Los meses de invierno: junio y agosto presentaron los valores mínimos con montos de 0.0 y $26.5 \mathrm{~mm}$ respectivamente. La razón de estos valores bajo se debe al congelamiento del estanque (evaporímetro) por las temperaturas negativas que suceden en esta estación. Un detalle de los montos mensuales de esta variable se muestra en la última columna de la tabla 3.

E: INSOLACIÓN Y NUBOSIDAD. Durante 2012 se registró un total de 1.711 horas y 15 minutos de sol, cantidad inferior en 33 horas y 5 minutos al total registrado durante el 2011. El mes con mayor insolación fue febrero con 230 horas y 25 minutos de sol, seguido de enero con 208 horas de sol. Otros meses con más de 160 horas de sol fueron octubre, noviembre, y diciembre. Por su parte, los meses de menor insolación correspondieron a julio y junio con: 76 horas y 20 minutos; 76 horas y 30 minutos respectivamente de sol; cabe destacar que estos dos meses representan a la estación de invierno que es donde la posición del sol está más alejada de la tierra (movimiento de traslación) y los ángulos de incidencia son más pequeños lo que trae como consecuencia la escasas horas de sol en esta estación para la latitud $53^{\circ}$ Sur.

El promedio anual de nubosidad fue de 5.9 octavos de cielo cubierto, valor superior en 0.1 octavos al promedio registrado en el año 2011. El mayor promedio mensual se registró en abril con un valor de 6.4 octavos de cielo, seguido de febrero y noviembre, mientras que los menores promedios se registraron en los meses de junio y marzo. Un
Tabla 5. Insolación y nubosidad.

\begin{tabular}{lccc}
\hline Meses & \multicolumn{2}{c}{$\begin{array}{l}\text { Horas y } \\
\text { minutos }\end{array}$} & Octavos \\
\hline Enero & 208 & $: 0$ & 6.3 \\
Febrero & 230 & $: 25$ & 5.8 \\
Marzo & 144 & $: 0$ & 5.5 \\
Abril & 122 & $: 40$ & 6.4 \\
Mayo & 86 & $: 45$ & 5.7 \\
Junio & 76 & $: 30$ & 5.4 \\
Julio & 76 & $: 20$ & 6.0 \\
Agosto & 118 & $: 0$ & 5.7 \\
Septiembre & 133 & $: 0$ & 5.8 \\
Octubre & 177 & $: 45$ & 5.8 \\
Noviembre & 172 & $: 30$ & 6.3 \\
Diciembre & 165 & $: 20$ & 6.2 \\
\hline Total & 1711 & $: 15$ & 5.9 \\
\hline
\end{tabular}

detalle mensual de la insolación y la nubosidad se muestra en la tabla 5.

F. RADIACIÓN SOLAR GLOBAL. Durante el año 2012, esta radiación promedio anual alcanzó a los $12.5 \mathrm{Ly} / \mathrm{h}$, equivalentes a $145.8 \mathrm{Wm}^{-2}$. En comparación con el año 2011, este promedio fue superior en apenas $0.1 \mathrm{Ly} / \mathrm{h}$ mismo valor que corresponde al promedio anual normal. El mayor valor medio mensual lo registró diciembre, con un promedio de $22.0 \mathrm{Ly} / \mathrm{h}$, seguido de enero y noviembre con valores medios de $18.0 \mathrm{Ly} / \mathrm{h}$ y $17.5 \mathrm{Ly} / \mathrm{h}$ respectivamente. El resto de los meses no superaron los $16.0 \mathrm{Ly} / \mathrm{h}$. Entre los meses de menor radiación destacan los comprendidos entre mayo y agosto con promedios inferiores a $8.5 \mathrm{Ly} / \mathrm{h}$, obteniéndose los mínimos valores durante julio y junio: $4.0 \mathrm{Ly} / \mathrm{h}$ y 4.0 Ly/h, respectivamente.

Cabe destacar que los promedios mas altos se encuentran en la estaciones de primavera y verano; a su vez la estación de invierno presenta los promedios más bajos del año, esto en directa relación con el movimiento de traslación que efectúa la tierra durante un año solar medio.

En relación al año anterior, todos los promedios mensuales son casi similares y no presentan variaciones significativas importantes tanto positivas como negativas; esto se ve reflejado en la mínima diferencia 
de sólo $0.1 \mathrm{Ly} / \mathrm{h}$ en el promedio anual de este parámetro.

Con respecto a la variación diaria y a sus promedios mensuales, la distribución es bien definida y claramente estacional. Hay un aumento de la radiación hacia las horas del mediodía y hacia la estación de verano. El mayor promedio de radiación se alcanzó en enero y diciembre entre las 12 y las 14 horas con un valor promedio bi-horario de 54.0 Ly/h para ambos meses, de igual manera es posible encontrar valores altos entre las 10 y 16 horas.

Referente a la variación diaria de la radiación en su promedio anual y en comparación con el 2011, no se produjeron grandes variaciones de los promedios

TABLA 6. Radiación Solar Ly/h).

\begin{tabular}{lccccccccccccc} 
Hora & Ene. & Feb. & Mar. & Abr. & May. & Jun. & Jul. & Ago. & Sep. & Oct. & Nov. & Dic. & Año \\
\hline $00-02$ & 0.0 & 0.0 & 0.0 & 0.0 & 0.0 & 0.0 & 0.0 & 0.0 & 0.0 & 0.0 & 0.0 & 0.0 & 0.0 \\
$02-04$ & 0.0 & 0.0 & 0.0 & 0.0 & 0.0 & 0.0 & 0.0 & 0.0 & 0.0 & 0.0 & 0.0 & 0.0 & 0.0 \\
$04-06$ & 0.0 & 0.0 & 0.0 & 0.0 & 0.0 & 0.0 & 0.0 & 0.0 & 0.0 & 0.0 & 0.0 & 0.0 & 0.0 \\
$06-08$ & 12.0 & 18.0 & 6.0 & 0.0 & 0.0 & 0.0 & 0.0 & 6.0 & 6.0 & 12.0 & 18.0 & 18.0 & 8.0 \\
$08-10$ & 24.0 & 30.0 & 24.0 & 18.0 & 6.0 & 6.0 & 6.0 & 18.0 & 24.0 & 30.0 & 30.0 & 36.0 & 21.0 \\
$10-12$ & 36.0 & 42.0 & 42.0 & 30.0 & 18.0 & 18.0 & 12.0 & 24.0 & 36.0 & 42.0 & 42.0 & 48.0 & 32.5 \\
$12-14$ & 54.0 & 48.0 & 42.0 & 30.0 & 18.0 & 12.0 & 12.0 & 24.0 & 36.0 & 42.0 & 42.0 & 54.0 & 34.5 \\
$14-16$ & 42.0 & 36.0 & 36.0 & 24.0 & 12.0 & 12.0 & 12.0 & 18.0 & 30.0 & 36.0 & 42.0 & 48.0 & 29.0 \\
$16-18$ & 30.0 & 18.0 & 24.0 & 12.0 & 6.0 & 6.0 & 6.0 & 12.0 & 18.0 & 18.0 & 24.0 & 36.0 & 17.5 \\
$18-20$ & 18.0 & 12.0 & 12.0 & 6.0 & 0.0 & 0.0 & 0.0 & 0.0 & 6.0 & 6.0 & 12.0 & 18.0 & 7.5 \\
$20-22$ & 0.0 & 0.0 & 0.0 & 0.0 & 0.0 & 0.0 & 0.0 & 0.0 & 0.0 & 0.0 & 0.0 & 6.0 & 0.5 \\
$22-24$ & 0.0 & 0.0 & 0.0 & 0.0 & 0.0 & 0.0 & 0.0 & 0.0 & 0.0 & 0.0 & 0.0 & 0.0 & 0.0 \\
\hline Promedio & 18.0 & 17.0 & 15.5 & 10.0 & 5.0 & 4.5 & 4.0 & 8.5 & 13.0 & 15.5 & 17.5 & 22.0 & 12.5 \\
\hline
\end{tabular}

Nota: $\quad 1 \mathrm{Ly}=1 \mathrm{cal} / \mathrm{cm}^{2}=4.1868$ Joule $/ \mathrm{cm}^{2} .1$ Joule $=1$

entre las 10 y 22 horas. El resto presentó valores similares, incluido las horas comprendidas entre las 0 y las $4 \mathrm{~h}$ y entre las 22 y $24 \mathrm{~h}$, periodo en que la radiación fue nula. Los promedios bi-horarios de radiación global por meses y anual se muestran en la tabla 6.

G. VIENTOS. El promedio de velocidad para el 2012, a una altura de $10 \mathrm{~m}$ s.n.s, fue de $5.2 \mathrm{~m} / \mathrm{s}$, valor equivalente a $18.7 \mathrm{~km} / \mathrm{h}$.

Los meses más ventosos fueron octubre, marzo y noviembre con valores medios de $6.3 \mathrm{~m} / \mathrm{s} ; 5.9 \mathrm{~m} / \mathrm{s}$ y $5.7 \mathrm{~m} / \mathrm{s}$ respectivamente. Cabe destacar que nueve de doce meses presentaron promedios superiores a los $5 \mathrm{~m} / \mathrm{s}$. Los meses de más calma fueron los de otoño e invierno, entre los que se destaca mayo con una media de $4.1 \mathrm{~m} / \mathrm{s}$; agosto y abril con valores de
$4.5 \mathrm{~m} / \mathrm{s}$ y $4.7 \mathrm{~m} / \mathrm{s}$ respectivamente.

Al comparar estos valores con lo sucedido el año 2011, se observa una leve baja en el promedio anual de este meteoro en $0.4 \mathrm{~m} / \mathrm{s}$.

En la variación diaria en cada mes, los mayores promedios ocurrieron en horas del mediodía y comienzos de la tarde de los meses de septiembre, octubre y noviembre, mostrando una relación casi directa con los valores máximos de radiación solar para estas horas. El máximo promedio horario se registró entre las 11 y las 13 horas de octubre llegando a los $7.6 \mathrm{~m} / \mathrm{s}$. El mínimo en cambio, ocurrió entre las 23 y las 44 horas en mayo, con valores que apenas alcanzaron los $2.5 \mathrm{~m} / \mathrm{s}$.

Los promedios horarios anuales fueron máximos entre las 11 y 16 horas, con valores sobre 
TABLA 7. Viento promedio diario $(\mathrm{m} / \mathrm{s})$.

\begin{tabular}{|c|c|c|c|c|c|c|c|c|c|c|c|c|c|}
\hline Hora & Ene & Feb & Mar & Abr & May & Jun & Jul & Ago & Sep & Oct & Nov & Dic & Año \\
\hline $0-1$ & 4.3 & 3.6 & 4.7 & 3.6 & 3.0 & 3.1 & 3.5 & 3.8 & 4.1 & 4.8 & 4.1 & 4.5 & 3.9 \\
\hline $1-2$ & 4.5 & 3.8 & 4.7 & 3.2 & 2.8 & 3.3 & 3.0 & 3.4 & 4.1 & 5.1 & 4.0 & 3.8 & 3.8 \\
\hline $2-3$ & 4.7 & 4.0 & 4.7 & 3.2 & 3.0 & 3.5 & 3.2 & 3.1 & 4.4 & 5.5 & 4.3 & 3.6 & 3.9 \\
\hline 3- 4 & 4.6 & 4.2 & 4.8 & 3.4 & 3.4 & 3.9 & 3.5 & 3.3 & 4.8 & 5.7 & 4.5 & 3.6 & 4.1 \\
\hline 4- 5 & 4.8 & 4.2 & 5.0 & 3.7 & 4.0 & 4.3 & 3.8 & 3.2 & 5.2 & 5.8 & 5.0 & 3.6 & 4.4 \\
\hline 5- 6 & 4.9 & 4.4 & 5.2 & 4.0 & 4.5 & 4.7 & 4.1 & 3.5 & 5.8 & 6.0 & 5.5 & 3.6 & 4.7 \\
\hline 6- 7 & 5.1 & 4.6 & 5.4 & 4.4 & 5.0 & 5.1 & 4.7 & 3.7 & 6.1 & 6.2 & 5.6 & 3.8 & 5.0 \\
\hline 7- 8 & 5.4 & 4.8 & 5.6 & 4.3 & 5.2 & 5.2 & 5.3 & 3.6 & 6.2 & 6.2 & 5.7 & 3.9 & 5.1 \\
\hline 8- 9 & 5.7 & 5.3 & 5.8 & 4.8 & 5.0 & 5.2 & 5.9 & 4.1 & 6.3 & 6.4 & 5.7 & 4.3 & 5.4 \\
\hline $9-10$ & 5.8 & 5.5 & 5.9 & 4.8 & 4.9 & 5.1 & 6.3 & 4.5 & 6.4 & 6.7 & 6.1 & 4.4 & 5.5 \\
\hline $10-11$ & 5.7 & 5.3 & 6.1 & 5.2 & 4.9 & 5.6 & 6.9 & 4.6 & 6.8 & 7.0 & 6.1 & 4.5 & 5.7 \\
\hline $11-12$ & 5.8 & 5.8 & 6.1 & 5.7 & 4.8 & 6.2 & 7.0 & 5.0 & 6.7 & 7.6 & 6.5 & 4.4 & 6.0 \\
\hline $12-13$ & 5.8 & 6.2 & 6.3 & 5.6 & 4.7 & 6.9 & 6.5 & 5.2 & 6.7 & 7.6 & 6.7 & 4.9 & 6.1 \\
\hline $13-14$ & 5.9 & 6.2 & 6.4 & 5.9 & 4.6 & 7.3 & 6.7 & 5.4 & 7.3 & 7.4 & 6.9 & 4.9 & 6.2 \\
\hline $14-15$ & 5.7 & 6.6 & 6.4 & 6.0 & 4.8 & 7.4 & 6.9 & 5.6 & 6.8 & 7.3 & 6.9 & 5.0 & 6.3 \\
\hline $15-16$ & 5.6 & 6.5 & 6.7 & 6.3 & 4.9 & 7.0 & 6.8 & 5.3 & 6.7 & 7.4 & 6.8 & 5.2 & 6.3 \\
\hline $16-17$ & 5.3 & 6.2 & 7.0 & 6.0 & 4.7 & 6.8 & 6.8 & 5.3 & 6.3 & 7.5 & 6.8 & 5.1 & 6.2 \\
\hline $17-18$ & 5.2 & 6.0 & 7.3 & 5.9 & 4.6 & 6.6 & 6.3 & 5.5 & 6.0 & 7.1 & 6.4 & 5.3 & 6.0 \\
\hline $18-19$ & 5.0 & 5.4 & 7.1 & 5.6 & 4.1 & 5.7 & 6.1 & 5.6 & 5.3 & 6.8 & 6.1 & 5.1 & 5.7 \\
\hline $19-20$ & 4.8 & 5.0 & 6.9 & 5.1 & 3.9 & 5.4 & 5.7 & 5.0 & 5.1 & 5.8 & 5.8 & 5.4 & 5.3 \\
\hline $20-21$ & 4.5 & 4.8 & 6.3 & 4.8 & 3.6 & 4.5 & 5.5 & 5.1 & 4.6 & 5.7 & 5.4 & 5.5 & 5.0 \\
\hline $21-22$ & 4.0 & 4.1 & 6.0 & 4.4 & 3.3 & 3.7 & 4.9 & 4.6 & 4.3 & 5.3 & 5.0 & 5.9 & 4.6 \\
\hline $22-23$ & 4.3 & 4.0 & 5.5 & 4.3 & 2.8 & 3.5 & 4.6 & 4.4 & 4.3 & 5.2 & 4.6 & 6.0 & 4.5 \\
\hline 23-24 & 4.1 & 3.9 & 4.9 & 3.9 & 2.5 & 3.3 & 4.0 & 4.0 & 4.3 & 5.0 & 4.1 & 5.7 & 4.2 \\
\hline Media & 5.1 & 5.0 & 5.9 & 4.7 & 4.1 & 5.1 & 5.3 & 4.5 & 5.6 & 6.3 & 5.6 & 4.7 & 5.2 \\
\hline
\end{tabular}

los $6.0 \mathrm{~m} / \mathrm{s}$, mientras que las horas de más calma ocurrieron entre las 00 y las 03 horas, con valores inferiores o iguales a $3.8 \mathrm{~m} / \mathrm{s}$.

La tabla 7 muestra el detalle horario, mensual y anual de la velocidad media del viento.

De las velocidades máximas alcanzadas por el viento, la racha máxima ocurrió el 24 de julio a las $12: 15$ con dirección oeste (W) y alcanzó a los $31.9 \mathrm{~m} / \mathrm{s}$ (114.8 km/h). Otras rachas importantes ocurrieron en febrero, junio y agosto con valores de
$29.2 \mathrm{~m} / \mathrm{s}(105.1 \mathrm{~km} / \mathrm{h}) ; 29.3 \mathrm{~m} / \mathrm{s}(105.5 \mathrm{~km} / \mathrm{h}) \mathrm{y}$ $28.9 \mathrm{~m} / \mathrm{s}(104.0 \mathrm{~km} / \mathrm{h})$ respectivamente. En forma general, a excepción de mayo y septiembre, todos los meses presentaron rachas máximas de viento sobre los $20 \mathrm{~m} / \mathrm{s}(72.0 \mathrm{~km} / \mathrm{h})$. La tabla 8 muestra la racha máxima instantánea por mes.

De acuerdo a los valores de magnitud, se clasificaron estas velocidades en seis rangos que definen velocidades bajas a las: $0.5<\mathrm{v}<2.1 \mathrm{~m} / \mathrm{s} ; 2.1<\mathrm{v}<3.6 \mathrm{~m} / \mathrm{s}$, velocidades medias a: $3.6<\mathrm{v}<5.7 \mathrm{~m} / \mathrm{s} ; 5.7<\mathrm{v}$ 
TABLA 8: Rachas máximas $(\mathrm{m} / \mathrm{s})$ mensuales de viento por dirección.

\begin{tabular}{cccccccccccc}
\hline Ene & Feb & Mar & Abr & May & Jun & Jul & Ago & Sep & Oct & Nov & Dic \\
22.1 & 29.2 & 28.3 & 20.1 & 18.0 & 29.3 & 31.9 & 28.9 & 17.5 & 28.3 & 26.8 & 26.8 \\
\hline NW & NW & W & NW & W & W & W & W & NE & N & SW & W \\
\hline
\end{tabular}

$<8.8 \mathrm{~m} / \mathrm{s}$ y velocidades altas para: $\mathrm{v}>8.8 \mathrm{~m} / \mathrm{s}$ y $11.1 \mathrm{~m} / \mathrm{s}$. Durante el 2012 las velocidades bajas representaron el $30.2 \%$ del tiempo, mientras que las velocidades medias completaron un $56.7 \%$ del tiempo y las velocidades altas representaron un $13.1 \%$ del total.

La tabla 9 muestra la frecuencia relativa del viento por cada dirección y rango de velocidad; y el porcentaje total de observaciones por dirección. Se observa que los vientos predominantes fueron mayoritariamente del oeste (W) $52.2 \%$ del total de observaciones, seguido de las direcciones noroeste (NW) con un $18.5 \%$ y el norte con un $10.3 \%$ de las observaciones. Los vientos llamados westerlies (Schneider et. al. 2003, Endlicher \& Santana 1988, Weischet 1985) suman el $77.0 \%$ del total de tiempo con vientos en la ciudad. Las horas de calmas netas alcanzaron al $0.16 \%$ del tiempo. Las direcciones restantes mostraron porcentajes pequeños.

En forma anual, para el 2012 las velocidades altas (v>8.8 m/s) ocurrieron de la dirección oeste, alcanzando un $10.4 \%$ del tiempo. Otras direcciones que mostraron velocidades altas fueron el noroeste y el norte. Las otras direcciones mostraron porcentajes de estas velocidades inferiores al $1 \%$; destacándose las del componente este, noreste y sureste.

La rosa de los vientos de la figura 1 muestra

Tabla n9: Frecuencia de velocidad y dirección del viento. Punta Arenas. 2012.

\begin{tabular}{ccccccccc}
\hline Direc./vel $(\mathrm{m} / \mathrm{s})$ & $0.5-2.1$ & $2.1-3.6$ & $3.6-5.7$ & $5.7-8.8$ & $8.8-11.1$ & $>=11.1$ & Total & $\%$ \\
\hline $\mathrm{N}$ & 277 & 107 & 286 & 202 & 22 & 9 & 902 & 10.3 \\
$\mathrm{NE}$ & 128 & 35 & 95 & 64 & 3 & 0 & 324 & 3.7 \\
$\mathrm{E}$ & 95 & 26 & 85 & 59 & 4 & 3 & 270 & 3.1 \\
$\mathrm{SE}$ & 86 & 29 & 59 & 42 & 2 & 0 & 218 & 2.5 \\
$\mathrm{~S}$ & 140 & 26 & 82 & 45 & 11 & 4 & 307 & 3.5 \\
$\mathrm{SW}$ & 164 & 64 & 152 & 143 & 20 & 2 & 545 & 6.2 \\
$\mathrm{~W}$ & 679 & 245 & 962 & 1780 & 713 & 199 & 4577 & 52.2 \\
$\mathrm{NW}$ & 413 & 138 & 423 & 490 & 122 & 38 & 1623 & 18.5 \\
Total & 1980 & 669 & 2142 & 2824 & 896 & 255 & 8764 & 100 \\
$\%$ & 22.6 & 7.6 & 24.4 & 32.2 & 10.2 & 2.9 & 100 & \\
\hline
\end{tabular}

las direcciones del viento y seis rangos de velocidad para cada una de éstas. En cada dirección se puede medir el porcentaje de horas con respecto al total registrado durante el año y a la vez se puede medir, en porcentaje, el rango en que el viento sopló en cada dirección.

\section{AGRADECIMIENTOS}

Se agradece a las siguientes personas $e$ Instituciones que colaboraran en hacer posible este resumen meteorológico. Al Sr. Rodrigo Ojeda O. por su labor como observador meteorológico; al personal de la Dirección General de Aguas (DGA), 


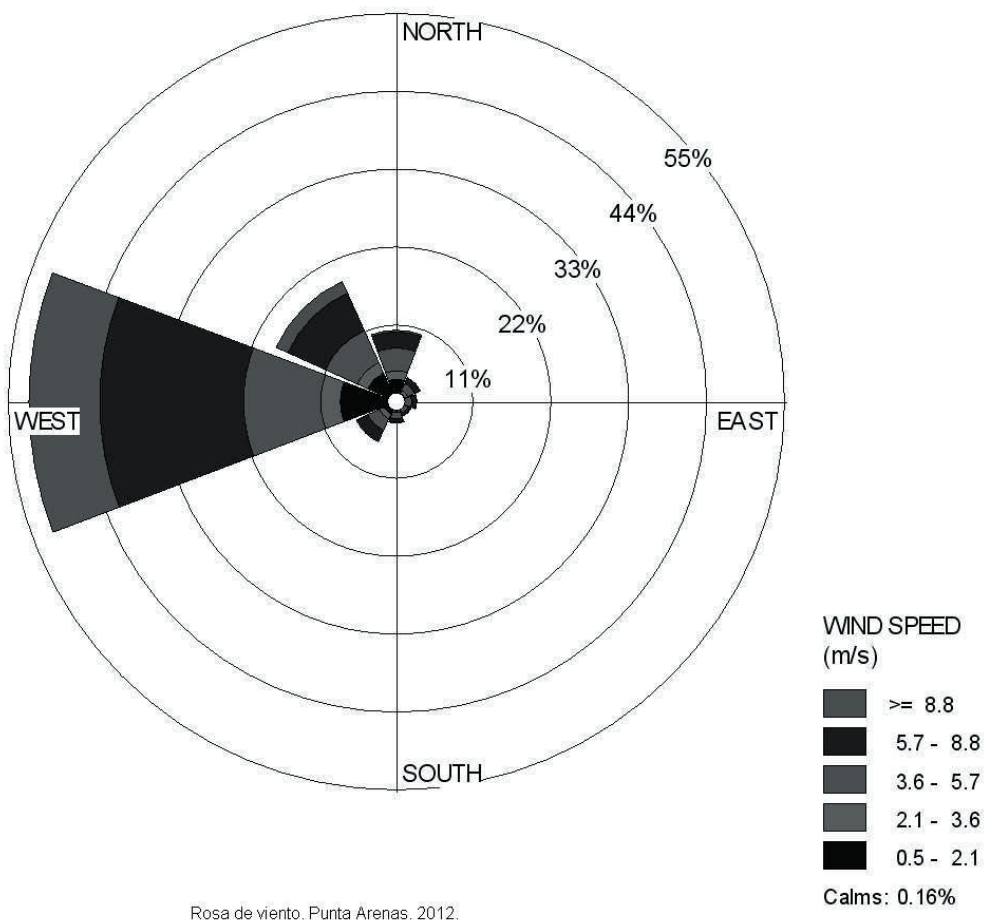

Fig., 1. Rosa de los vientos de ocho direcciones y tres rangos de velocidad para el año 2012.

que en forma constante velan por el mantenimiento y suministro necesario de insumos propios para el funcionamiento normal de la estación climática. A la Patagonia Research Fundation (PRF) representada por el señor Charles Porter, por la disposición de una estación automática de tiempo (AWS) complementando el instrumental de la estación Jorge Schythe. A la DMC (Dirección Meteorológica de Chile) por su apoyo en suministros a la estación y al Sr. Alex Stowhas Suárez por la digitalización y procesamiento en los datos de viento.

\section{LITERATURA CITADA}

Butorovic N. (2012).Resumen Meteorológico año 2011. Estación Jorge C. Schythe. Anales Instituto Patagonia (Chile), 40(1), 173-182.

Endlicher W. \& Santana A. (1988). El clima del sur de la Patagonia y sus aspectos ecológicos. Un siglo de mediciones climatológicas en Punta Arenas. Anales Instituto Patagonia
Serie Cs.Nat. (Chile) 18, 57-86.

Santana A., A. (1984). Variación de las precipitaciones de 97 años en Punta Arenas como indice de posibles cambios climáticos. Anales Instituto Patagonia Serie Cs. Nat. (Chile) 15, 51-60.

Santana A., Butorovic N. \& Olave C. (2009). Variación de la temperatura en Punta Arenas (Chile) en los últimos 120 años. Anales Instituto Patagonia (Chile), 37(1), 85-96.

Schneider, C., Glaser M., Kilian R., Santana A., Butorovic N. \& Cassassa G. (2003). Weather Observations Across the Southern Andes at $53^{\circ}$ S. Physical Geography, 24(2), 97-119.

Weischet, W. (1985). Climatic constraits for the development of the Far South of Latin America.

Geojournal, 11(1), 79-87.

Zamora, E. \& A. Santana (1979). Oscilaciones y tendencias térmicas en Punta Arenas entre 1888 y 1979 . Anales Instituto Patagonia (Chile) 10, 147-154. 